\title{
Executive dysfunction in autism
}

\author{
Elisabeth L. Hill \\ Department of Psychology, Goldsmiths College, University of London, Whitehead Building, New Cross, \\ London, SE14 6NW, UK \\ Corresponding author: Elisabeth L. Hill (E.hill@gold.ac.uk).
}

\begin{abstract}
'Executive function' is an umbrella term for functions such as planning, working memory, impulse control, inhibition and mental flexibility, as well as for the initiation and monitoring of action. The primacy of executive dysfunction in autism is a topic of much debate, as are recent attempts to examine subtypes of executive function within autism and other neurodevelopmental disorders that are considered to implicate frontal lobe function. This article will review cognitive behavioural studies of planning, mental flexibility and inhibition in autism. It is concluded that more detailed research is needed to fractionate the executive system in autism by assessing a wide range of executive functions as well as their neuroanatomical correlates in the same individuals across the lifespan.
\end{abstract}


'Executive function' is traditionally used as an umbrella term for functions such as planning, working memory, impulse control, inhibition and shifting set as well as the initiation and monitoring of action [1]. These functions share the need to disengage from the immediate environment to guide actions. Executive functions are typically impaired in patients with acquired damage to the frontal lobes as well as in a range of neurodevelopmental disorders that are likely to involve congenital deficits in the frontal lobes. Such clinical disorders include attention deficit hyperactivity disorder (ADHD), obsessive compulsive disorder, Tourette syndrome, phenylketonuria, schizophrenia and autism spectrum disorder. It should be noted that executive dysfunction can be observed in those with acquired damage to non-frontal brain areas.

Autism spectrum disorder is a developmental disorder characterised by impaired social interaction and communication as well as repetitive behaviours and restricted interests. It is a lifelong disorder and affects at least $0.6 \%$ of the population with males being affected three times more often than females [2,3]. Estimates of learning disability vary. In a recent review of epidemiological studies the percentage of individuals with a learning disability is given as $70 \%$ [4]. Although different labels are used to define people at each end of the spectrum (e.g. autism, Asperger syndrome), 'autism' will be used in this review to refer to the whole spectrum.

In recent years three key cognitive theories and their variants have been investigated in an attempt to understand the link between the brain and behaviour in autism. The most well-known of these is the theory of mind deficit hypothesis [5-10], and another account is that of weak central coherence [11-15] (see Box 1). Although these two accounts together can explain many of the deficits and assets associated with autism, repetitive behaviours and restricted interests might best be explained by a third cognitive theory: that of executive dysfunction.

\section{Executive dysfunction in autism}

Over the past twenty years several executive functions have been studied in autism. In this review, I focus on three of these: planning, flexibility and inhibition (see [16-18] for detailed reviews of these and other aspects of executive function in autism).

\section{Planning}

Planning is a complex, dynamic operation in which a sequence of planned actions must be constantly monitored, re-evaluated and updated.Children, adolescents and adults with autism have been reported to be impaired on planning tests,suchastheTower ofLondon(seeBox2).This is the case in relation to age-matched clinical control groups including dyslexia, ADHD and Tourette syndrome [19-24] as well as to age-matched normally developing individuals [21] (see Figure 1). Furthermore this impairment is maintained over time [23]. (For detailed information of studies of planning, see Supplementary Table $1_{1}$ and at http:// archive.bmn.com/supp/tics/Hill_Suppl_tables.pdf)

An elegant computerised study that uses a variant of the Tower of London, the 'Stockings of Cambridge' [25] (see Box 2), sheds some light on the specific aspects of difficulty on this planning task. Performance of children and adolescents with autism was compared with that of two control groups, one equivalent to the autistic group for both age and (moderate) learning disability and the other a younger normally developing control group selected to match the approximate verbal and non-verbal mental ages of the autistic participants. The autistic group was impaired relative to both comparison groups. However, this planning deficit was evident only on puzzles requiring a longer sequence of moves. Furthermore, success was positively related to non-verbal mental age. This finding suggests that general ability per se has at least some influence on successful planning abilities. Autism might be a contributing factor, indeed autism plus learning disability could lead to an additive deficit. 


\section{Mental flexibility}

Poor mental flexibility is illustrated by perseverative, stereotyped behaviour and difficulties in the regulation and modulation of motor acts. This indicates problems in the ability to shift to a different thought or action according to changes in a situation. One task in which poor mental flexibility has been shown in autism is theWisconsin Card Sorting Task (WCST; [26-28] and see Box 2). On this and similar tasks, individuals with autism experience a difficulty in mental flexibility in relation to normally developing individuals and those with other neurodevelopmental disorders [24,25] (Figure 2). The majority of studies have focused on perseverative responses, that is where the shift to sorting by a new rule is not easily made. Studies evaluating several measures have consistently found deficits in the total number of categories correctly identified and the total number of errors made. The deficit on the WCST is maintained over time [23] and is not restrictedtoWesterncultures [26]. (Fordetailedinformation of studies of flexibility see Supplementary Table 2 and at http://archive.bmn.com/supp/tics/Hill_Suppl_tables.pdf)

Using the the Intradimensional-Extradimensional shift (ID/ED shift) task of the CANTAB (see Box 2) children and adolescents with autism have been evaluated at different levels of flexibility in comparison with control groups matched for either age and (moderate) learning disability or for approximate verbal and non-verbal mental age. The autistic group was impaired in comparison with both of the control groups only in the final stages of the task, that is when an extradimensional shift was required [25]. This study suggests that it is not that autistic individuals perseverate in a global sense, rather that they experience an autism-specific 'stuck-in-set' perseveration.

\section{Inhibition}

The story regarding inhibition is somewhat different [29-35]. On a classic test of inhibition, the Stroop task (see Box 2), autistic children and adolescents are unimpaired [21,24,36] (Figure 2). This is in contrast to other neurodevelopmental disorders clearly associated with executive dysfunction such as ADHD [21] and phenylketonuria [37]. Furthermore, autistic performance is normal on some other tests of inhibition, in particular a test of negative priming ([31]; Box 2) and neutral inhibition conditions of a Go/No-Go task ([30]; Box 2). Prepotent inhibition and cognitive flexibility conditions of a Go/No-Go task were impaired in relation to age, gender and IQ matched normally developing individuals [30]. Autistic children have also been shown to perseverate significantly more than a mental age matched control group on theWindows and Detour-Reaching tasks [32-35] (Box 2). This difficulty is seen in situations with and without a social component, as well as when the rules of the 'game' are both arbitrary or nonarbitrary.

Overall, these findings have been taken to indicate a difficulty in the inhibition of a prepotent response. However, as performance on the Stroop test - involving the inhibition of a prepotent response - is unimpaired in autism, how can these results be reconciled? Russell has postulated that the rules of some executive function tests appear arbitrary to autistic individuals and that it is this that causes the observed difficulties. He argues that executive function tests that do not lack a rationale are passed by those with autism[38]. (For detailed information of studies of inhibition see Supplementary Table $3_{1}$ and at http://archive.bmn.com/supp/tics/Hill_Suppl_tables.pdf)

\section{Lifespan perspectives}

With the realization that frontal lobe development can be seen in young children, rather than only from later childhood as was once thought [39], studies of executive function in preschool children have emerged. Given the complexity of tasks of executive function used with older children and adults, it is not possible to make comparisons using the well-known tasks described above. Using tests developed for nonhuman primates as well as for young children, studies of preschool-aged autistic children have identified a pattern of intact and impaired performance in executive 
functioning [40-43]. The greatest deficits being seen in the older samples and on a test of mental flexibility. However, further potential group differences are likely to have been masked by floor and ceiling effects [42]. (For detailed information of studies of executive function in young autistic children see Supplementary Table 41 and at http://archive.bmn.com/supp/tics/Hill_Suppl_tables.pdf)

Linking executive function ability with a key deficit in young children with autism, these studies have focused on the possible relationship between joint attention and executive functions in autism. Joint attention develops rapidly from 6 to 12 months and involves the sharing of attention between the infant, another person and an object or event (e.g. looking at mother while pointing to a toy to share enjoyment). Joint attention is almost totally universal in autism and is an early indicator of the disorder [44]. Deficits in mental flexibility but not in the on-line maintenance and manipulation of information (taken as another executive function), were seen in young children with autism of approximately four years in relation to developmentally disordered as well as normally developing controls. Twelve months later joint attention impairment at the first test was a significant predictor of mental flexibility (assessed by a spatial reversal task) at test two in the autism group only [43].

The link between joint attention and executive function has been investigated further in studies that have drawn particular parallels between behavioural tasks and neuroanatomical structures. Dawson and colleagues have studied young autistic children in comparison with normal developing children and those with Down syndrome. Although performance of autistic 34-year-olds was impaired on tasks associated with ventromedial (VMPFC) and dorsolateral (DLPFC) prefrontal areas, only performance on the former was found to be correlated strongly with joint attention ability [40,41]. At five years, difficulties were observed on delayed response and delayed non-matching to sample tasks, both tasks associated with VMPFC, once non-verbal mental ability had been covaried [40]. Structural equationmodelling indicated that performance on these tasks wasmore strongly associated with joint attention abilities than performance on tasks associated with activation of DLPFC, suggesting that core autism symptoms might be related specifically to VMPFC [40].

\section{Neuroanatomical considerations}

\section{Structural abnormalities in frontal cortex in autism}

Executive function is a cognitive construct used to describe behaviours thought to be mediated by the frontal lobes. So what evidence is there of neuroanatomical dysfunction in the frontal lobes in autism? Structural studies of the autistic brain are limited, although several cortical and subcortical abnormalities have been identified [45]. Transient delayed postnatal maturation of the frontal lobes [46], serotinergic abnormalities in prefrontal cortex [47] and structural abnormality in orbitofrontal cortex [48] have all been reported.

\section{Relating performance to neuroanatomical structures}

Neuroanatomical correlates of performance on tests of executive function have not yet been widely investigated in autism although reduced activation of DLPFC has been described in a spatial working memory task [49]. Furthermore, parallels have been made from behavioural performance on tasks associated with specific areas of prefrontal cortex [40,41]. Detailed functional imaging studies of tasks of executive function, as well as investigations of brain structure, will be crucial if we are to understand more appropriately the extent, nature and cause of executive dysfunction in the autist, and the implications of such dysfunction.

Several explanations for the link between brain and behaviour have been raised in relation to autism and executive dysfunction. One of these has hypothesized executive dysfunction to be a consequence of abnormalities in medial temporal lobe (MTL) function, with variability 
in performance on executive tasks arising from the varying severity of MTL brain abnormalities [50]. Two versions of this account place executive dysfunction as associated to, rather than causal to the core autistic symptoms. By this account, impairments in prefrontal function in older individuals with autism are secondary consequences of early MTL dysfunction, consequences that do not become apparent until the frontal lobes mature [50].

An alternative view is that a more direct link exists between frontal lobe abnormality and executive dysfunction. Autistic performance might be tied to dysfunctional integration of the frontal lobes with the rest of the brain, abnormal developments in neuronal sophistication and/or abnormal myelinisation (see, [49,51]). Findings of transient delayed postnatal maturation of the frontal lobes in autism $[46,52]$ and reduced functional connectivity of frontal cortex with other cortical and subcortical regions (see, [49]) support this view. The failure of the frontal lobes to follow a normal maturational pattern is likely to have long-term consequences for all development. This abnormality might be reflected differentially over time as the impact of abnormal development on that of other connected systems is seen. Clearly further study is needed, and in particular longitudinal studies tracking participants over their lifespan using comparable tests.

\section{Further concerns}

A number of further issues can be raised after reading the literature concerning executive functions in autism. These include task complexity, the specificity of executive dysfunction to the autism spectrum, the universality of the findings across the spectrum, the influence of IQ and neuroanatomical correlates on behavioural performance. I will now discuss the first three issues, which have not been raised elsewhere in this review. Other questions for future research are highlighted in Box 3.

\section{Task complexity as an explanation of autistic performance?}

First, task complexity can confuse the interpretation of results. Tasks such as the WCST are multicomponent processes and cannot be considered to represent only one aspect of executive processing. Clearly flexibility, inhibition, working memory and monitoring are involved. Thus a detailed investigation of the many executive components of such tasks must be investigated within the same participant groups. Models for this can be seen in the stepwise nature of the ID/ED task [25] and the inhibition tasks adopted by Ozonoff and colleagues

[30,31]. Furthermore, task presentation can be crucial to performance in autistic individuals. Computerised presentation of tasks - where the social component is reduced - attenuates performance, but does not remove the deficit [53]. By comparing traditional and computerised task presentations it will be possible to identify how independent of one another executive and social abnormalities are in autism.

\section{Executive dysfunction as a diagnostic marker for autism?}

Could executive dysfunction be a diagnostic marker for autism? One hindrance is the fact that executive dysfunction is found in neurodevelopmental disorders other than autism. However studies have generally reported a specific pattern of executive dysfunction that distinguishes autism from such other disorders including ADHD, Tourette syndome and conduct disorder $[16,21,22]$. Particularly important is the differing executive profile of those with autism versus ADHD, a disorder in which clear deficits in inhibition are seen and lesser deficits in planning $[16,22]$. Continued, detailed investigation of executive functions in different clinical populations are needed. If a specific but differential profile of executive dysfunction is identified in these disorders then the possibility arises of using measures of executive function as a marker for the diagnosis of autism. 


\section{The broader autism phenotype}

The possibility of using one or more measures of executive functioning as a diagnostic marker for autism is coming closer with the findings of executive dysfunction in the broader autism phenotype. Essentially, a broader cognitive phenotype exists when biological relatives of an autistic individual show a raised incidence of cognitive performance associated with the diagnosis of autism, but to a mild degree that does not obtain them a diagnosis. A small number of studies have been conducted in the biological parents and siblings of autistic

probands, suggesting that deficits in planning and flexibility characterize the broader phentoype [54-57]. Dawson and colleagues have argued recently that executive function is one of six candidate autism broader phenotype measures for the focus of genetic studies [50]. Integrating genetic, brain and behavioural perspectives must be key for future progress in the understanding of autism.

\section{The universality of executive dysfunction to autism}

For the executive dysfunction account of autism to be valid as a cognitive account of the primary symptoms, these difficulties must be a universal feature of autism, that is they must be a characteristic of every affected individual. To-date, a handful of studies have found that their tests of executive function have not identified deficits in participants [58-61], although this might simply be a function of the measures selected for these studies and thus the universality of executive dysfunction in autism cannot yet be ruled out. Studies focusing on a range of executive functions, as well as those reflecting naturalistic situations need to be investigated. Not only will this help to clarify the universality of executive dysfunction to autism, but also the impact of social aspects of tasks that could cause increased difficulty in autism. Of additional benefit will be an empirical demonstration of the ways in which different aspects of executive performance correlate and fractionate in autism and whether this maps clearly onto the patterns seen in the normal population. Recent investigations along these lines in normal children and adults have identified three dimensions of executive functions, which have been characterised as relating to: (i) inhibition, (ii) flexibility, and (iii) working memory (or updating) $[62,63]$.

\section{Conclusion}

Our understanding of executive dysfunction in autism has progressed over the past decade and it has been possible to identify components of intact executive function as well as dysfunction in the disorder. Overall, school-aged and adult autistic individuals of all ability ranges are impaired in the executive function of planning and show a certain type of perseverative behaviour, taken to indicate a deficit in mental flexibility. These individuals do not exhibit impaired inhibitory control per se, although they do show impaired inhibition of a prepotent response in certain cases, perhaps reflecting the forced application of an arbitrary rule. Progress can be made by considering performance on a wide range of tasks with clear stages of progression and tightly matched control tasks in wellmatched control groups. A clearer conceptualisation of how executive dysfunction develops across the lifespan of autistic individuals will be beneficial. By adopting these approaches in future research, advances in our understanding of executive function in the autistic and nonautistic brain should follow, including detailed development of theoretical accounts. Most importantly this will lead to significant improvements in the methods that we can implement to ameliorate the consequences of executive dysfunction in the daily lives of individuals with autism spectrum disorder.

\section{Acknowledgements}

This work was completed while the author was at the Institute of Cognitive Neuroscience, University College London. It was supported

by fundingfromtheMedicalResearchCouncil(UK)toUtaFrith (grantnumber G9716841) and 
facilitated by the MRC Cooperative at the Institute of CognitiveNeuroscience,UCL.Mythanks tomembers of the Developmental Disorders and Executive Functions research groups at the Institute of Cognitive Neuroscience, in particular to Uta Frith, Sarah White and Chris Bird.

\section{References}

1 Stuss, D.T., Knight, R.T. eds, (2002) Principles of Frontal Lobe Function, Oxford University Press

2 Baird, G. et al. (2000) A screening instrument for autism at 18 months of age: a 6-year follow-up study. J. Am. Acad. Child Adolesc. Psychiatry 39, 694-702

3 Chakrabarti, S. and Fombonne, E. (2001) Pervasive developmental disorders in preschool children.

J.A.M.A. 285, 3093-3099

4 Fombonne, E. (2003) Epidemiological surveys of autism and other pervasive developmental disorders: an update. J. Autism Dev. Disord. 33, 365-382

5 Frith, U. and Frith, C.D. (2003) Development and neurophysiology of mentalizing. Philos. Trans. R. Soc. Lond. B Biol. Sci. 358, 459-473

6 Frith, U. (2003) Autism. Explaining the Enigma, 2nd edn, Blackwell

7 Baron-Cohen, S. et al. (1985) Does the autistic child have a 'theory of mind'? Cognition 21, 37-46

8 Gallagher, H.L. and Frith, C.D. (2003) Functional imaging of 'theory of mind'. Trends Cogn. Sci. 7, 77-83

9 Happe', F. et al. (1996) 'Theory of mind' in the brain. Evidence from a PET scan study of Asperger syndrome. Neuroreport 8, 197-201

10 Castelli, F. et al. (2002) Autism, Asperger syndrome and brain mechanisms for the attribution of mental states to animated shapes. Brain 125, 1839-1849

11 Happe', F. (1999) Autism: cognitive deficit or cognitive style? Trends Cogn. Sci. 3, 216-222

12 Mottron, L. et al. (2000) Local and global processing of music in highfunctioning persons with autism: beyond central coherence? J. Child Psychol. Psychiatry 41, 1057-1065

13 Plaisted, K. (2001) Reduced generalization in autism: an alternative to weak central coherence. In The Development of Autism: Perspectives from Theory and Research (Burack, J.A. et al., eds), pp. 149-169, Erlbaum

14 Ring,H.A. et al. (1999) Cerebral correlates of preserved cognitive skills in autism: a functional MRI study of embeded figures task performance. Brain 122, 1305-1315

15 Frith, C.D. (2003) What do imaging studies tell us about the neural basis of autism? In Autism: Neural Basis and Treatment Possibilities (Rutter, M., ed.), John Wiley and Sons

16 Pennington, B.F. and Ozonoff, S. (1996) Executive functions and developmental psychopathology. J. Child Psychol. Psychiatry 37, 51-87

17 Russell, J. (1997) How executive disorders can bring about an adequate 'theory of mind.'. In Autism as an Executive Disorder (Russell, J., ed.), pp. 256-304, Oxford University Press

18 Hill, E.L. Evaluating the theory of executive dysfunction in autism. Dev. Rev. (in press).

19 Ozonoff, S. et al. (1991) Executive function deficits in high-functioning autistic individuals: relationship to theory of mind. J. Child Psychol. Psychiatry 32, 1081-1105

20 Bennetto, L. et al. (1996) Intact and impaired memory functions in autism. Child Dev. 67, 1816-1835 
21 Ozonoff, S. and Jensen, J. (1999) Brief report: specific executive function profiles in three neurodevelopmental disorders. J. Autism Dev. Disord. 29, 171-177

22 Sergeant, J.A. et al. (2002) How specific is a deficit of executive functioning for attentiondeficit/hyperactivity disorder? Behav. Brain Res. 130, 3-28

23 Ozonoff, S. and McEvoy, R.E. (1994) A longitudinal study of executive function and theory of mind development in autism. Dev. Psychopathol. 6, 415-431

24 Ozonoff, S. (1997) Components of executive function in autism and other disorders. In Autism as an Executive Disorder (Russell, J., ed.), pp. 179-211, Oxford University Press

25 Hughes, C. et al. (1994) Evidence for executive dysfunction in autism. Neuropsychologia 32, 477-492

$26 \mathrm{Shu}, \mathrm{B}-\mathrm{C}$. et al. (2001) Executive function deficits in non-retarded autistic children. Autism 5, 165-174

27 Nelson, H.E. (1976) A modified card sorting test sensitive to frontal lobe defects. Cortex 12, 313-324

28 Heaton, R.K. et al. (1993) Wisconsin Card Sorting Test Manual: Revised and Expanded, Psychological Assessment Resources

29 Stroop, J.R. (1935) Studies of interference in serial verbal reactions. J. Exp. Psychol. 18, 643-662

30 Ozonoff, S. et al. (1994) Executive function abilities in autism and Tourette syndrome: an informationprocessing approach. J. Child Psychol. Psychiatry 35, 1015-1032

31 Ozonoff, S. and Strayer, D.L. (1997) Inhibitory function in nonretarded children with autism. J. Autism Dev. Disord. 27, 59-77

32 Russell, J. et al. (1991) The 'Windows task' as a measure of strategic deception in preschoolers and autistic subjects. Br. J. Dev. Psychol. 9, 101-119

33 Russell, J. et al. (2003) Mechanising an executive task: the performance of preschool children, children with autism and with moderate learning difficulties in the automated Windows Task. Cogn. Dev. 18, 111-137

34 Hughes, C. and Russell, J. (1993) Autistic children's difficulty with mental disengagement from an object: its implications for theories of autism. Dev. Psychol. 29, 498-510

35 Bý'ro, S. and Russell, J. (2001) The execution of arbitrary procedures by children with autism. Dev. Psychopathol. 13, 97-110

36 Russell, J. et al. (1999) Two intact executive capacities in children with autism: implications for the core executive dysfunctions in the disorder. J. Autism Dev. Disord. 29, 103-112

37 Diamond, A. et al. (1997) Prefrontal cortex cognitive deficits in children treated early and continuously for PKU. Monogr. Soc. Res. Child Dev. 62, 1-208

38 Russell, J. (2002) Cognitive theories of autism. In Cognitive Deficits in Brain Disorders (Harrison, J.E. and Owen, A.M., eds), pp. 295-323, Martin Dunitz

39 Mesulam, M-M. (2002) The human frontal lobes: transcending the default mode through contingent encoding. In Principles of Frontal Lobe Function (Stuss, D.T. and Knight, R.T., eds), pp. 8-30,

Oxford University Press

40 Dawson, G. et al. (1998) Neuropsychological correlates of early symptoms of autism. Child Dev. 69, 12761285

41 Dawson, G. et al. (2002) Neurocognitive function and joint attention ability in young children with autism spectrum disorder versus developmental delay. Child Dev. 73, 345-358

42 McEvoy, R.E. et al. (1993) Executive function and social communication deficits in young autistic children. J. Child Psychol. Psychiatry 34, 563-578 
43 Griffith, E.M. et al. (1999) Executive functions in young children with autism. Child Dev. 70, 817-832 44 Osterling, J. and Dawson, G. (1994) Early recognition of children with autism: a study of first birthday home videotapes. J. Autism Dev. Disord. 24, 247-257

45 Kemper, K.L. and Bauman, M.L. (1998) Neuropathology of infantile autism. J. Neuropathol. Exp. Neurol. $57,645-652$

46 Zilbovicius, M. et al. (1995) Delayed maturation of the frontal cortex in childhood autism. Am. J. Psychiatry $152,248-252$

47 Chugani, D.C. et al. (1997) Altered serotonin synthesis in the dentatothalamocortical pathway in autistic boys. Ann. Neurol. 42, 666-669

48 Salmond, C.H. et al. (2003) Investigating individual differences in brain abnormalities in autism. Philos. Trans. R. Soc. Lond. B Biol. Sci. 358, 405-413

49 Luna, B. et al. (2002) Neocortical system abnormalities in autism. An fMRI study of spatial working memory. Neurology 59, 834-840

50 Dawson, G. et al. (2002) Defining the broader phenotype of autism: genetic, brain, and behavioral perspectives. Dev. Psychopathol. 14, 581-611

51 Chugani, D.C. (1998) A critical period of brain development: studies of cerebral glucose utilization with PET. Preventative Medicine 27, 184-188

52 Ohnishi, T. et al. (2000) Abnormal regional cerebral blood flow in childhood autism. Brain 123, 18381844

53 Ozonoff, S. (1995) Reliability and validity of theWisconsin card sorting test in studies of autism. Neuropsychology 9, 491-500

54 Ozonoff, S. et al. (1993) Can standard measures identify subclinical markers of autism. J. Autism Dev. Disord. 23, 429-441

55 Piven, J. and Palmer, P. (1997) Cognitive deficits in parents from multiple-incidence autism families. J. Child Psychol. Psychiatry 38, 1011-1021

56 Hughes, C. et al. (1997) Executive function in parents of children with autism. Psychol. Med. 27, 209-220

57 Hughes, C. et al. (1999) Towards a cognitive phenotype for autism: increased prevalence of executive dysfunction and superior spatial span amongst siblings of children with autism. J. Child Psychol. Psychiatry 40, 705-718

58 Minshew, N.J. et al. (1992) Neuropsychological functioning in nonmentally retarded autistic individuals. J. Clin. Exp. Neuropsychol. 14, 749-761

59 Russell, J. and Hill, E.L. (2001) Action-monitoring and intention reporting in children with autism. J. Child Psychol. Psychiatry 42, 317-328

6o Hill, E.L. and Russell, J. (2002) Action memory and self-monitoring in children with autism: self versus other. Infant and Child Development 11, 159-170

61 Baron-Cohen, S. et al. (1999) A mathematician, a physicist and a computer scientist with Asperger syndrome: performance on psychology and folk physics tests. Neurocase $5,475-483$

62 Miyake, A. et al. (2000) The unity and diversity of executive functions and their contributions to complex 'frontal lobe' tasks: a latent variable analysis. Cogn. Psychol. 41, 49-100

63 Lehto, J. et al. (2003) Dimensions of executive functioning: evidence from children. Br. J. Dev. Psychol. 21, 59-80 


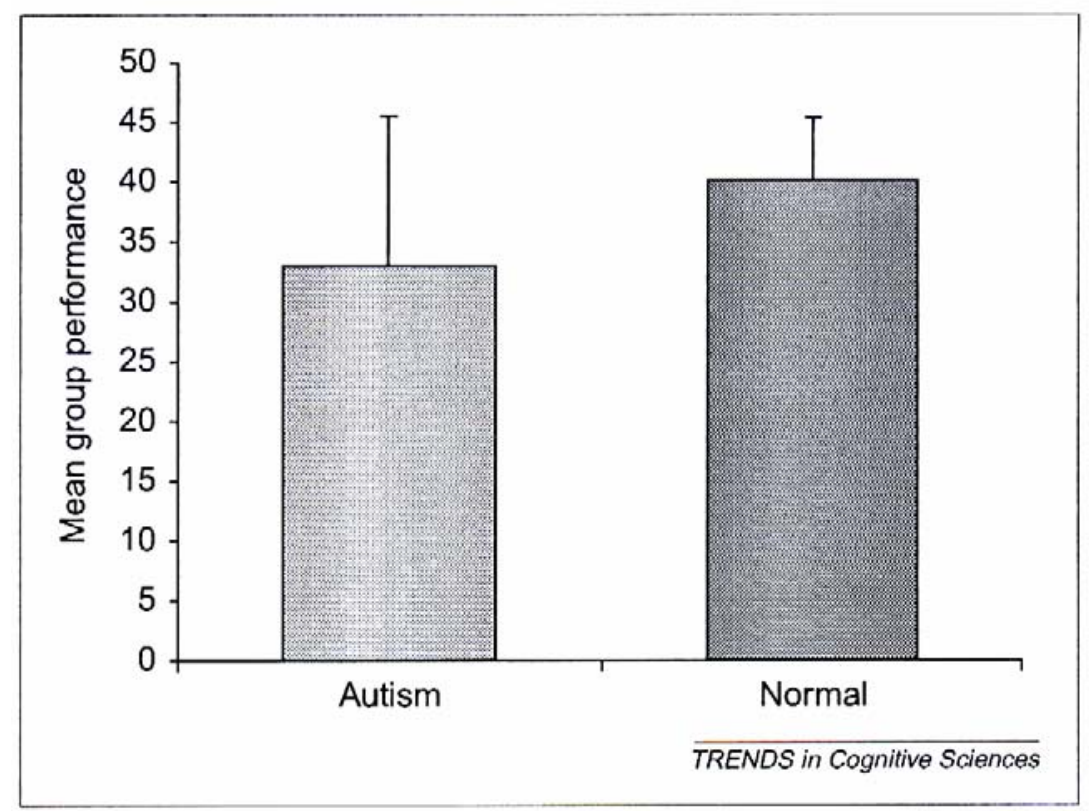

Figure 1. Mean group performance of children with autism and normally developing children on the Tower of Hanoi task. Groups were matched for chronological age. Nine puzzles (requiring 1 to 5 moves) were completed. The performance of children with autism was significantly worse than that of the normally developing children $(p<0.01)$. Data redrawn from [21].

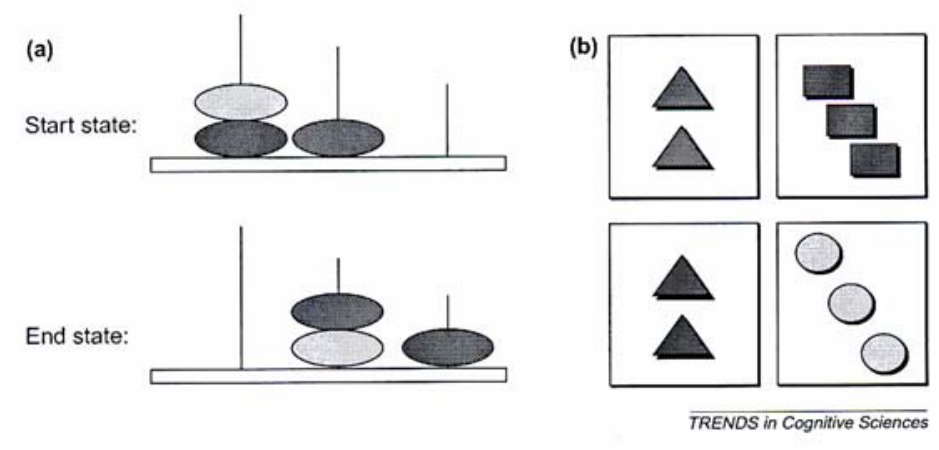

Figure L. (a) Example of a puzzle used in the ToL task. Discs must be moved from the start state to the end state in as few moves as possible. (b) Examples of cards used in the WCST. Cards can be sorted by colour, number or shape, depending on what the current 'rule' is. 


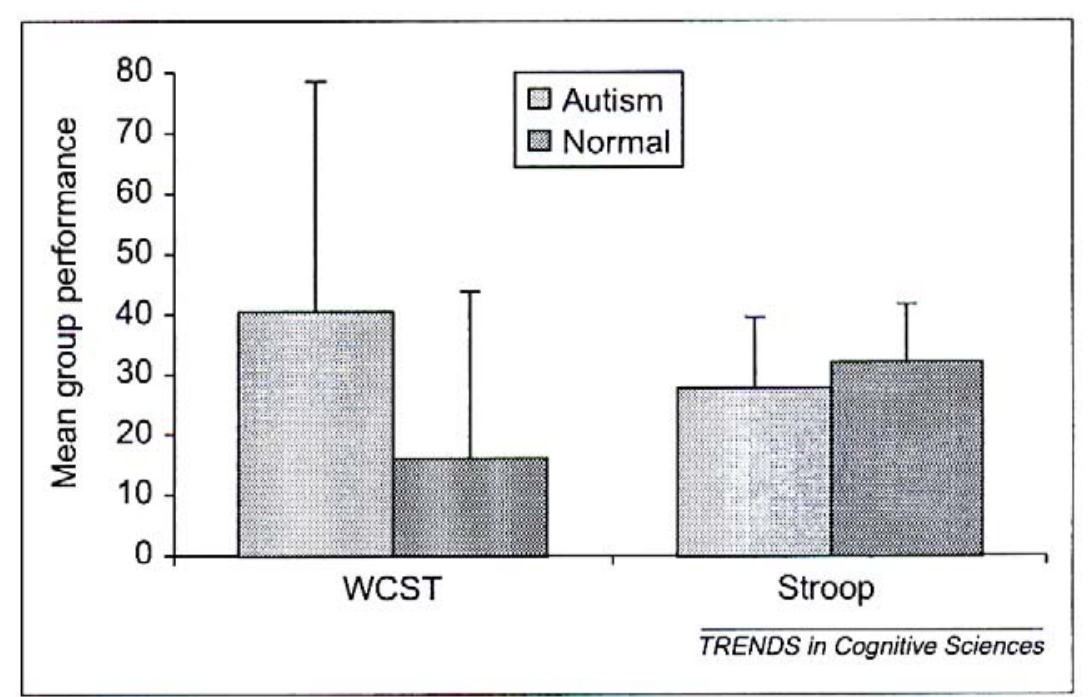

Figure 2. Mean group performance of children with autism (blue) and normally developing children (red). Groups were matched for chronological age. The performance of children with autism was significantly worse than that of the normally developing children on the WCST ( $p<0.01$ ), but not on the Stroop test (high scores indicate poorer performance). Data redrawn from |21|. (See also Supplementary information online ${ }^{1}$ and at: http://archive.bmn.com/supp/tics/ Hill...Suppl...tables.pdf).

\section{Box 1. Two cognitive theories of autism spectrum disorder \\ Theory of mind}

Theory of mind, or mentalising, refers to the ability to identify, attribute and manipulate mental states such as beliefs and desires. This abilitydevelops rapidly inyoung children [5], but at an extremely slow rate in autism [6]. A widely used test of theory of mind is the 'Sally-Anne test' [7]. Neuroimaging studies have identified a network of brain regions active during mentalising. This network centres on the anterior paracingulate cortex, the superior temporal sulci and the temporal poles bilaterally [8].Whenundertaking mentalisingtasks, individuals with Asperger syndrome show significantly less activation in the brain regions important for mentalising in normal individuals $[9,10]$.

Weak central coherence

Autism is characterised by a series of strengths as well as weaknesses. Tests that tap factual knowledge and focussed attention on detail can lead to peak performances, whereas tests tapping common sense comprehension can be surprisingly poor. Some of these features are explained by the theory of 'central coherence'. This account refers to an information processing style, rather than a deficit. This cognitive style relates to the tendency to process incoming information in its context [11]. In the case of strong central coherence this tendency would work at the expense of attention to and memory for details. In the case of weak central coherence, piecemeal processing is favoured at the expense of contextual meaning. For example, when retelling a story typical individuals find it easier to recall accurately the gist of the story rather than its specific details. People with autism show the opposite profile. Thus, individuals with autism are described as exhibiting 'weak central coherence'.

An important extension of the central coherence account postulates not poor integration of information in a gestalt, but rather enhanced discrimination of individual elements [12,13].

The brain basis of central coherence has been little explored (but see [14]), although preliminary suggestions are that the early stages of sensory processing (where emphasis is paid to the local features of a stimulus) are intact in autism whereas the top-down modulation of these early processing stages (requiring the extraction of the global features of a stimulus) is not functioning appropriately [15]. 
Box 2. Executive tests in autism

Planning

The typical task used to assess planning is the Tower of Hanoi, or the related Tower of London (ToL) task, in which individuals must move disks from a prearranged sequence on three different pegs to match a goal state determined by the examiner (see Figure Ia). This must be done in as few moves as possible and following several specific rules. Puzzles are designed to be completed in two or more moves, increasing the level of difficulty of the task. Computer presentation of the task allows planning and motor execution times to be computed.

The Tower of Hanoi task is identical to the ToL except that the disks vary in size and the pegs on which they are placed are each an identical size. In the Stockings of Cambridge - part of the computerised Cambridge Neuropsychological Automated Battery (CANTAB) - the disks are suspended in inverted, sock-like holders.

\section{Mental flexibility}

The Wisconsin Card Sorting test (WCST; $[27,28]$ ) is generally interpreted as a test of mental flexibility (or setshifting). In this task an individual must sort cards (see Figure Ib) on one of three possible dimensions (colour, number, shape) according to a non-spoken rule, and is then required to shift rules to sort cards along a different dimension. The experimenter tells the participant whether s/he has placed the card correctly (i.e. followed the correct rule), but does not give the participant the rule explicitly. A wide range of different measures can be used for scoring this test including total number of errors, number of perseverative errors and categories completed. Here a perseverative error is viewed as a failure to shift set to the new sorting criterion.

A similar test of mental flexibility is the Intradimensional-Extradimensional shift (ID/ED shift) task of the CANTAB [25]. This task is presented in several stages, providing a more precise identification of the locus of difficulty than is possible using the WCST, and allowing participation by a wider range of individuals. In the early stages of the test, discriminationmust be made between one of two pink shapes, and in later stages between one of four stimuli (two pink shapes and two white lines). In the final stages of the test, shifts in discrimination are required within (ID) and between (ED) set (pink shapes and white lines respectively).

Inhibition

The classic Stroop test [29] is a well-known test of inhibition. In this task a participant must first read a list of colour names written in black ink (or name a series of blocks of colour) and then read a list of colour names written in coloured ink, where the ink colour is congruent with the colour word (e.g. 'blue' written in blue ink). Finally a participant must name the colour ink that colour words arewritten in (e.g. say 'red' to the word blue written in red ink). In this task the interference of one input (in this case the word) can bemeasured on the performance of another (naming the ink colour).

Ozonoff and colleagues have developed a range of tests of inhibition to elucidate the performance profile of autistic individuals. These include Go/No-Go [30], negative priming [31] and Stop-Signal [31] tasks. In the Go/No-Go task, participants were presented with a circle and triangle on a computer screen. In the first, 'neutral inhibition' task condition, participants must respond always to the same ('go') stimulus, either the circle or the triangle. In the second, 'prepotent inhibition' condition, a participant's 'go' stimulus was the opposite of the one in the neutral inhibition condition. In the final, 'flexibility condition', participants must shift from one response pattern to another, as the 'go' stimulus was frequently altered as determined by the computer.

Tests of negative priming assess an individual's level of interference when a previous target becomes the distractor item on a subsequent trial. In their negative priming task, Ozonoff and Strayer showed participants a string of five letters, such as FTFTF, and asked them to judge whether letters two and four were the same or different. Respondents were typically slower and less accurate to make a response when a letter in position two or four was shown in positions one, three and five on the previous trial.

In their Stop-Signal task, a test of categorisation, Ozonoff and Strayer required participants to categorise words as either animals, non-animals or words that had been predetermined as forbidden. One button was pressed if the presented word was an animal, and another button if the presented word was either a non-animal or a forbidden word. A subset of words were presented in conjunction with an auditory signal. No response was to be made when the auditory signal was heard, irrespective of the category that the word presented belonged to.

Russell and colleagues have developed two tests assessing the inhibition of a prepotent response. In the Windows and automated Windows tasks a participant can only win a desired object (a chocolate) by pointing away from this object $[32,33]$. In the Detour-Reaching tasks the participant can only reach inside a box for a ball by first making a rather unrelated action (e.g. flicking a switch) [34,35]. 
Box 3. Questions for future research

- What is the influence of IQ on executive performance in autism?

- What neural mechanisms correspond to specific executive functions in those with and without autism?

- What is the profile of executive function and dysfunction in autism across the lifespan?

- In those autistic individuals who show no clear executive deficits in laboratory tasks, how do they perform in naturalistic settings involving executive functions, such as shopping?

- Is there a specific profile of executive dysfunction unique to autism and distinct from other neurodevelopmental disorders associated with executive dysfunction?

- What would a cognitive model of executive functions in autism look like? 